\title{
Sensitivity of power spectral density noise techniques to numerical parameters in analyzing neutron noise experiments
}

\author{
E. Gilad ${ }^{\mathrm{a}, *}$, B. Geslot ${ }^{\mathrm{b}}$, P. Blaise ${ }^{\mathrm{b}}$, C. Dubi $^{\mathrm{c}}$ \\ ${ }^{a}$ The Unit of Nuclear Engineering, Ben-Gurion University of the Negev, Beer-Sheva 84105, Israel \\ ${ }^{b} \mathrm{DEN} / C A D / D E R / S P E x / L P E, C E A$ Cadarache, Saint-Paul-les-Durance 13108, France \\ ${ }^{c}$ Department of Physics, Nuclear Research Center NEGEV (NRCN), Beer-Sheva 84190, Israel
}

\begin{abstract}
Power spectral density methods (Cohn- $\alpha$ methods) are well-known and widely used for the analysis of neutron noise experiments and obtaining the reactor core integral kinetic parameters, i.e., the effective delayed neutron fraction $\beta_{\text {eff }}$ and the prompt neutron generation time $\Lambda$. The Cohn- $\alpha$ methods are considered as the standard data processing procedure in the case of a current acquisition system that works at high fission rates. Many uncertainties are usually considered in the Cohn- $\alpha$ method, e.g., statistical fluctuations in the neutron count, power drifts, uncertainties in the Diven factor, the integral fission rate, and in the reactivity value. However, the uncertainty associated with the numerical parameters used in the power spectra calculation procedure, e.g., time bin size and buffer size, is hardly discussed in the literature and generally overlooked.

In this paper, The Cohn- $\alpha$ method is implemented to analyze critical and subcritical configurations of the MAESTRO core in the MINERVE zero power reactor in order to measure its $\beta_{\text {eff }}$ and $\Lambda$ integral kinetic parameters. Both cross-correlation and auto-correlation power spectral densities are calculated and the kinetic parameters are obtained via Lorentzian curve fitting over the calculated spectra. The sensitivity of the obtained kinetic parameters to the choice of numerical parameters used for spectrum calculations is studied and found to be pronounced and comparable with other uncertainties. A novel methodology is proposed for analyzing the kinetic parameters' sensitivity to the power spectra calculations and for quantifying the associated uncertainties.

Keywords: Noise techniques, power spectral density, sensitivity analysis, integral kinetic parameters, spectrum calculations
\end{abstract}

\footnotetext{
*Corresponding author

Email address: gilade@bgu.ac.il (E. Gilad)
} 


\section{Introduction}

A set of neutron noise measurements have been performed on the MINERVE zero power reactor at Cadarache research center in France during September 2014. This experimental campaign was conducted in the framework of a tri-partite collaboration between CEA, PSI and SCK-CEN (Geslot et al., 2015; Perret, 2015; Gilad et al., 2016). Measurements were then also processed and analyzed in the framework of a collaboration between CEA, Ben-Gurion University of the Negev (BGU), and the Israeli Atomic Energy Commission (IAEC). The main purpose of the campaign was to obtain the core kinetic parameters using various existing and novel noise techniques and compare it with recent measurements. The last time a similar campaign was performed in MINERVE was in 1975 and the core configuration was different (Carre and Oliveira, 1975). This campaign is a continuation of a previous one aimed at determining the delayed neutron fraction $\beta_{\text {eff }}$ in the MINERVE reactor using in-pile oscillations technique (Gilad et al., 2015).

Several well-known and widely used neutron noise techniques were implemented for analyzing the experimental measurements, e.g., Cohn- $\alpha$, Feynman-Y, and Rossi- $\alpha$ methods. These methods were used to obtain the reactor core integral kinetic parameters, i.e., the effective delayed neutron fraction $\beta_{\text {eff }}$ and the prompt neutron generation time $\Lambda$ (Geslot et al., 2015; Gilad et al., 2016). More specifically, the Cohn- $\alpha$ method is considered as the standard data processing procedure in the case of a current acquisition system that works at high fission rates by digitizing the current signal issued by fission chambers (Diniz and dos Santos, 2002; dos Santos et al., 2006; Geslot et al., 2015). Such a system has recently been developed and qualified by CEA and is able to process signals on line without any data loss (de Izarra et al., 2015).

The statistical uncertainties associated with the Cohn- $\alpha$ method are usually thoroughly analyzed and are propagated to the final results, i.e., the integral kinetic parameters, using well established methodologies and considerations. For example, Geslot et al. (2015) recommend using the values obtained by the Cross-Correlation Power Spectral Density (CPSD) estimator following data processing considerations and final uncertainties associated with the results. This estimator has proved to be very robust and produced minimum uncertainties. The uncertainties usually considered in the Cohn- $\alpha$ method include statistical fluctuations in the neutron count, power drifts, uncertainties in the Diven factor, the integral fission rate, and in the reactivity value. The high-level analysis by Geslot et al. (2015) using Power Spectral Density (PSD) techniques leads to uncertainties of $1.8-2.8 \mathrm{pcm}$ in the value of $\beta_{\text {eff }}$ and $0.7-1.3 \mu \mathrm{s}$ in $\Lambda$ (at $1 \sigma$ ).

On the other hand, the uncertainty associated with the numerical parameters used in the power spectra calculation procedure, e.g., time bin size and buffer size, is hardly discussed in the literature and generally overlooked. Despite their conspicuous importance (as demonstrated in this paper), very little considerations are usually given to their values. These values are often determined rather arbitrarily according to the acquisition system technical specifications and the bias degree of the residuals in the curve fitting procedure. Moreover, well-defined criteria or methodologies for setting and tuning these numerical parameters, as well 
as for evaluating their associated uncertainties, are generally absent.

In this paper, the sensitivity of the Cohn- $\alpha$ method to numerical parameters used in the power spectra calculation procedure is studied by analyzing noise measurements performed in the MINERVE reactor core at three different reactivity states. The associated uncertainties are evaluated and a methodology for optimal determination of these parameters is proposed. The experimental setup is described in section 2 , the Cohn- $\alpha$ formalism is introduced in section 3, and the CPSD results for the critical state Acq12 are described and discussed in sections 4. The CPSD results for the subcritical states Acq16 and Acq19 are described in sections 4.4. APSD results for the different reactivity states are described section 4.5 and the conclusions are discussed in section 5 .

\section{Experimental setup}

The MINERVE reactor is a pool-type $\left(\sim 120 \mathrm{~m}^{3}\right)$ reactor operating at a maximum power of $100 \mathrm{~W}$ with a corresponding thermal flux of $10^{9} \mathrm{n} / \mathrm{cm}^{2} \cdot \mathrm{s}$ (Cacuci, 2010). The core is composed of a driver zone, which includes 40 standard highly enriched MTR-type metallic uranium alloy plate assemblies surrounded by a graphite reflector. An experimental cavity, in which various $\mathrm{UO}_{2}$ or MOX cladded fuel pins can be loaded in different lattices, reproducing various neutron spectra (Cacuci, 2010; pascal Hudelot et al., 2004), is located in the center of the driver zone. During the experimental campaign, the central experimental zone was loaded with $7703 \%$ enriched $\mathrm{UO}_{2}$ fuel rods arranged in a lattice representative of a PWR spectrum. An oscillator piston, capable of moving periodically and vertically between two positions located inside and outside of the core is located inside the experimental zone. A general view of the MINERVE reactor is shown in Fig. 1, together with schematic drawings of the reactor geometrical configuration and the MAESTRO core configuration (Leconte et al., 2013).

During the measurement campaign, neutron noise experiments have been conducted in three reactor states; one very close to critical state (marked as "Acq12") and two different subcritical states (marked as "Acq16" and "Acq19"). The different criticality states were obtained by inserting one of the four control rods into the core. The reactor configuration was that of the MAESTRO program (Leconte et al., 2013), representing a PWR spectrum in the central experimental cavity, as shown in Fig. 1. Two large fission chambers with approximately $1 \mathrm{~g}$ of ${ }^{235} \mathrm{U}$ have been installed next to the driver zone (denoted $\mathrm{n}^{\circ} 670$ and $\mathrm{n}^{\circ} 671$ in Fig. 1). In order to minimize flux disturbances in the detectors during measurement, reactor criticality was controlled by control rod B1, which is far from the two detectors. During the measurements, the power was regulated by an automatic piloting system that makes use of a low efficiency rotating control rod with cadmium sectors. The only slightly subcritical measurement Acq12 has been conducted at a power of $0.2 \mathrm{~W}$ with detectors' count rate around $5.5 \times 10^{5} \mathrm{cps}$. The subcritical measurements Acq16 and Acq19 have been conducted with detectors' count rate around $4 \times 10^{4} \mathrm{cps}$. The signals were acquired using fast 
amplifiers and CEA-developed multipurpose acquisition system X-MODE. The signals were acquired in time stamping mode with a resolution of 25 ns. A 1-second sample segment of the detectors' signal is shown in Fig. 2. More details on the experimental setup and acquisition systems can be found in (Geslot et al., 2015). The measurements analyzed in this paper are described in Table 1.

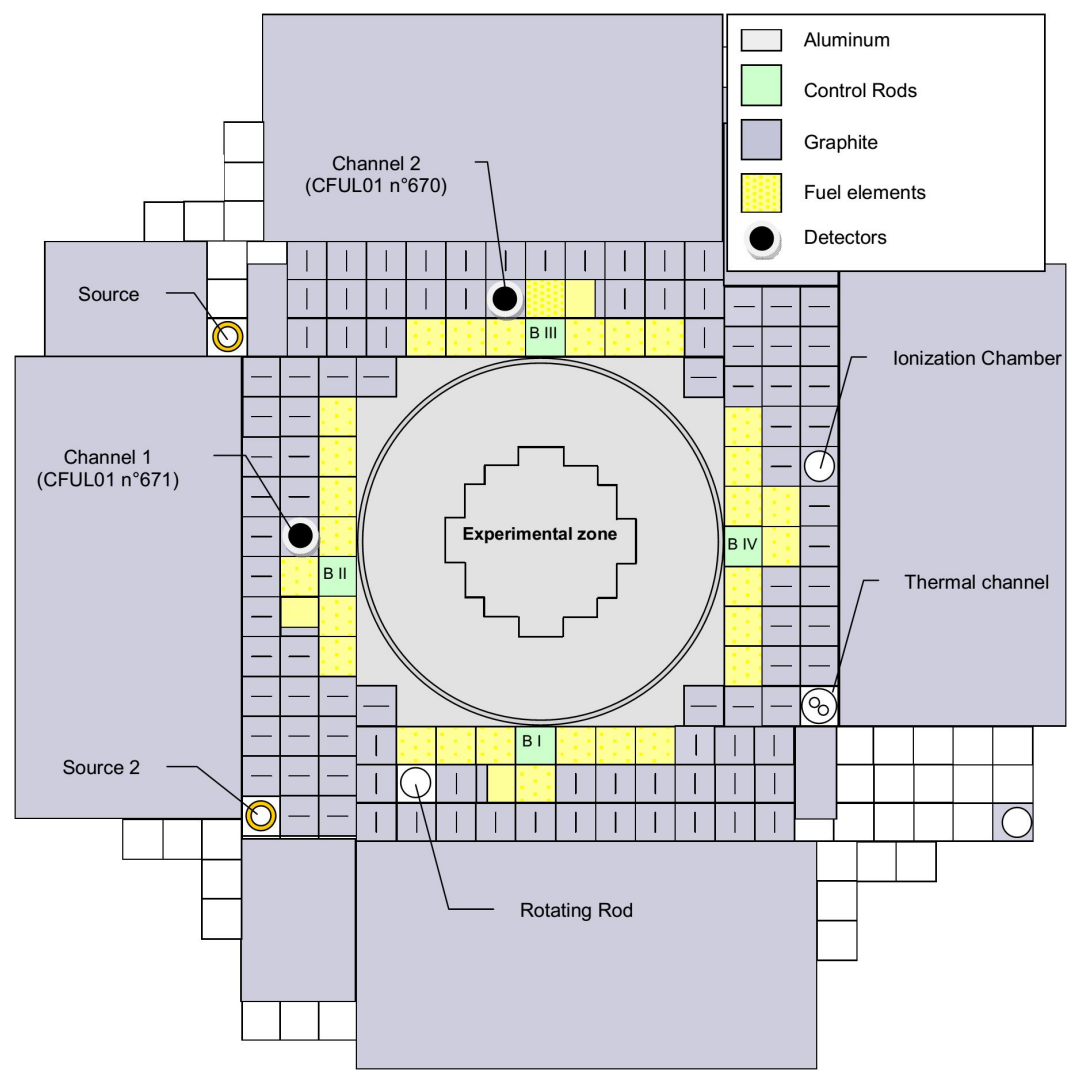

Figure 1: Schematic layout of the MINERVE zero power reactor core during the noise measurements campaign in Sep. 2014.

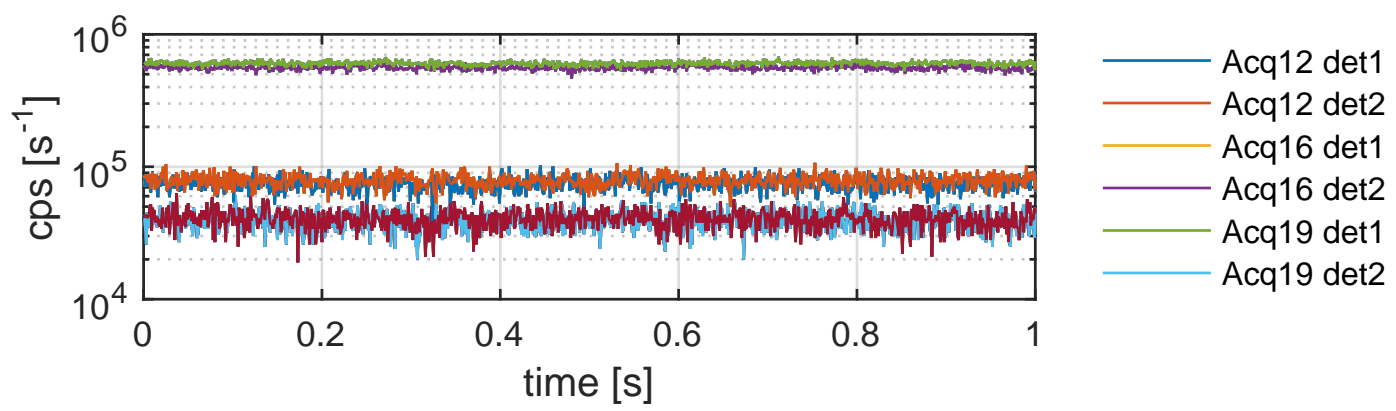

Figure 2: A sample segment of the detectors' signals for the different reactivity states of the core. 
Table 1: Pile noise measurements during the Sep. 2014 experimental campaign that are analyzed.

\begin{tabular}{llll}
\hline Data set & Acq12 & Acq16 & Acq19 \\
\hline Control rod height $[\mathrm{mm}]$ & B1@499 & B1@399 & B1@449 \\
Core power [W] & 0.2 & 0 & 0 \\
Duration [s] & 5400 & 5500 & 5500 \\
Integral fission rate $F\left[\mathrm{~s}^{-1}\right]$ & $6.45 \times 10^{9}$ & $4.00 \times 10^{8}$ & $7.91 \times 10^{8}$ \\
Reactivity [pcm] & $\sim 0$ & -230 & -117 \\
\hline
\end{tabular}

\section{The Cohn- $\alpha$ formalism}

The transfer function of the reactor links the reactor neutron population to the neutron source fluctuations. The zero power transfer function can be derived from point kinetic equations, where the source noise is considered to be entirely due to fluctuations in the core reactivity, in the neutron population and in the precursors concentration (Keepin, 1965; Williams, 1974; Santamarina et al., 2012). For large enough frequencies, i.e. $\omega \gg \lambda_{j}$, the transfer function amplitude takes the following form

$$
|H(\omega)|^{2}=\frac{\frac{1}{\left(\beta_{\text {eff }}-\rho\right)^{2}}}{1+\left(\omega / \omega_{c}\right)^{2}},
$$

where $\rho$ is the reactivity of the core and $\omega_{c}=\frac{\beta_{\text {eff }}-\rho}{\Lambda}$ is called the cutoff frequency.

The Cross-correlation Power Spectral Density (CPSD) is defined as the Fourier transform of the crosscorrelation between two detectors, i.e.,

$$
\mathrm{CPSD} \equiv \mathcal{F}\left\langle c_{1}(t), c_{2}(t)\right\rangle=\mathcal{F}\left\{c_{1}(t)\right\} \mathcal{F}^{*}\left\{c_{2}(t)\right\}=\mathcal{F}\left\{\frac{1}{2 T} \int_{-T}^{T} c_{1}(t) c_{2}(t+\tau) d t\right\},
$$

where $\mathcal{F}$ is the Fourier transform operator, $c_{i}(t)$ stands for the readings of detector $i$ as a function of time, $\langle x, y\rangle$ is the temporal correlation function between $x$ and $y, T$ represents the buffer size, $d t$ represents the time bin size, and $\mathcal{F}^{*}$ represents the complex conjugate of $\mathcal{F}$. Similarly, the Auto-correlation Power Spectral Density (APSD) is defined as the Fourier transform of the auto-correlation of a single detector $i$, i.e.

$$
\operatorname{APSD}_{i} \equiv \mathcal{F}\left\langle c_{i}(t), c_{i}(t)\right\rangle=\mathcal{F}\left\{c_{i}(t)\right\} \mathcal{F}^{*}\left\{c_{i}(t)\right\}=\mathcal{F}\left\{\frac{1}{2 T} \int_{-T}^{T} c_{i}(t) c_{i}(t+\tau) d t\right\} .
$$

The discrete form of the correlation function between two detectors $R_{12}(n)$ and its Fourier transform are as follows

$$
R_{12}(n)=\left\langle c_{1}, c_{2}\right\rangle(n)=\frac{1}{N-n} \sum_{k=0}^{N-n-1} c_{1, k} c_{2, k+n}
$$


and

$$
\operatorname{CPSD}(N, d t) \equiv \mathcal{F}\left\{R_{12}(n)\right\}=\sum_{k=0}^{N} \tilde{c}_{1, k} \tilde{c}_{2, k}^{*},
$$

where $N d t=T$ and $c_{x, k}$ and $\tilde{c}_{x, k}$ are the number of detections in detector $x$ in time bin $d t_{k}$ and its discrete Fourier transform, respectively, i.e., $\tilde{c}_{x, k}=\mathcal{F}\left\{c_{x, k}\right\}$. Similarly, the discrete form of the APSD of detector $i$ is

$$
\operatorname{APSD}_{i}(N, d t) \equiv \mathcal{F}\left\{R_{i i}(n)\right\}=\sum_{k=0}^{N} \tilde{c}_{i, k} \tilde{c}_{i, k}^{*} .
$$

The zero power transfer function amplitude $|H(\omega)|^{2}$ can also be written in terms of two detectors' readings $c_{1}(t)$ and $c_{2}(t)$ (Cohn, 1960; Santamarina et al., 2012) in the following form

$$
|H(\omega)|^{2}=\frac{\mathcal{F}\left\langle c_{1}(t), c_{2}(t)\right\rangle}{\bar{c}_{1} \bar{c}_{2}} \frac{1}{|\mathcal{F}(\delta \rho)|^{2}}=\frac{\mathrm{CPSD}}{\bar{c}_{1} \bar{c}_{2}} \frac{1}{2 D / F},
$$

where $\bar{c}_{i}$ is the average count rate of detector $i, \delta \rho$ is the reactivity perturbation, $D=\frac{\overline{\nu(\nu-1)}}{\bar{\nu}^{2}}$ is the Diven factor (Diven et al., 1956), and $F$ is the integral fission rate in the core. Hence, by combining Eqs. (1) and (7), the expression linking the CPSD with the effective delayed neutron fraction $\beta_{\text {eff }}$ and the prompt neutron generation time $\Lambda$ is (Cohn, 1960; Carre and Oliveira, 1975; Diniz and dos Santos, 2002; dos Santos et al., 2006) straightforward:

$$
2 \frac{D}{F} \frac{\frac{1}{\left(\beta_{\text {eff }}-\rho\right)^{2}}}{1+\left(\omega / \omega_{c}\right)^{2}}=\frac{\text { CPSD }}{\bar{c}_{1} \bar{c}_{2}} .
$$

Similarly, for the APSD, one gets:

$$
2 \frac{D}{F} \frac{\frac{1}{\left(\beta_{\text {eff }}-\rho\right)^{2}}}{1+\left(\omega / \omega_{c}\right)^{2}}=\frac{\operatorname{APSD}_{i}}{\bar{c}_{i} \bar{c}_{i}}+B_{i},
$$

where $B_{i}$ is some constant due to the fact that unlike CPSD, the APSD does not asymptotically tends to zero due to detections produced by the randomly (uncorrelated) arriving neutrons. In any case, for all practical purposes the RHS of Eqs. (8) and (9) is fitted with a function of the form

$$
f(\omega)=\frac{x_{1}}{1+\left(\frac{\omega}{x_{2}}\right)^{2}}+x_{3}
$$

and

$$
\beta_{\mathrm{eff}}-\rho=\sqrt{\frac{2 D}{F} \frac{1}{x_{1}}}, \quad \Lambda=\frac{\beta_{\mathrm{eff}}-\rho}{2 \pi x_{2}} .
$$

\section{Standard PSD analysis}

A total of three measurements were analyzed using the Cohn- $\alpha$ method (see Table 1). The Diven factor for thermal fission of ${ }^{235} \mathrm{U}$ is set to $D=0.8$. The integral fission rate $F$ is obtained by calculation of the flux distribution in the core and its calibration using the readings of a dedicated fission chamber located at the 
core center during the experiment. All measurements were recorded using the X-MODE acquisition system in time stamping mode with resolution of $25 \mathrm{~ns}$. The reactivity worth of the control rod B1 was calculated using rod-drop experiment and inverse kinetics analysis.

The standard Cohn- $\alpha$ procedure usually continues by evaluating the fission rate $F$ by calculation and measurement, calculating the CPSD in Eq. (5) from the measurement, and calculating the Diven factor $D$. Then, Eq. (8) is used to obtain $\beta_{\text {eff }}-\rho$ and the cutoff frequency $\omega_{c}=\frac{\beta_{\text {eff }}-\rho}{\Lambda}$ by Lorentzian curve fitting to the right hand size of the equation. An example for CPSD and APSD spectra for the different reactivity states and a fitted Lorentzian curve are shown in Fig. 3.
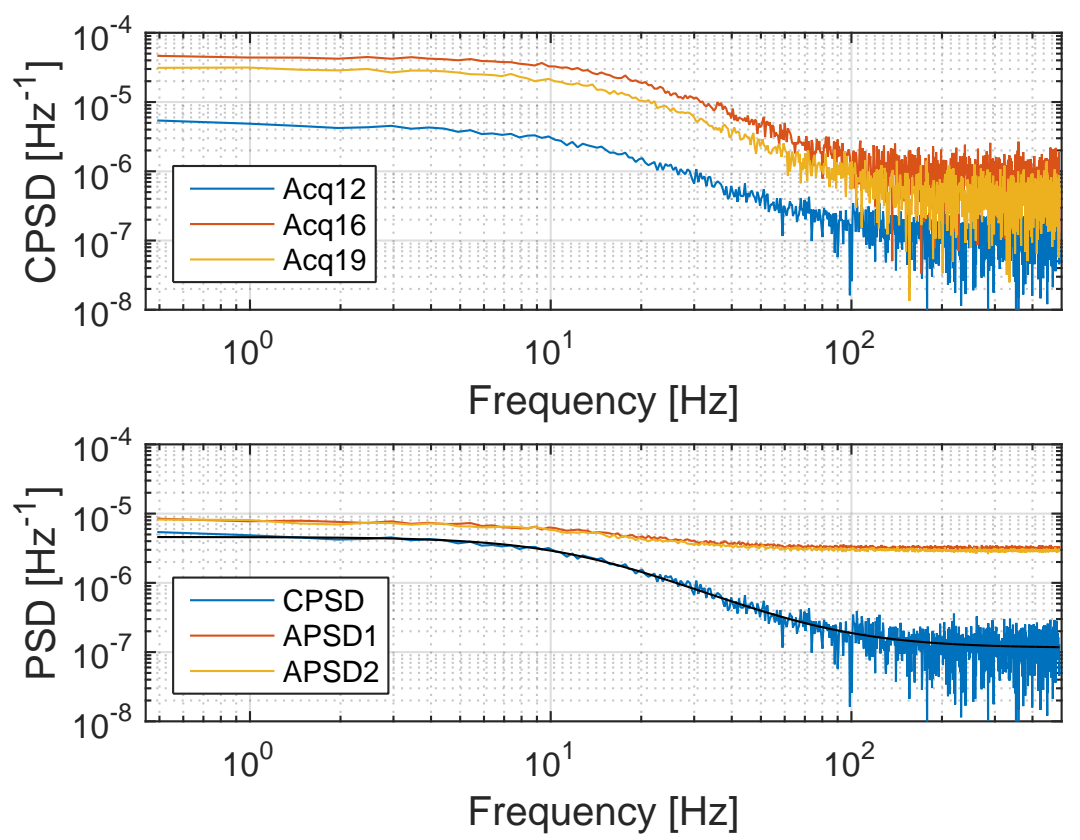

Figure 3: Upper panel: An example of CPSD spectra for the different reactivity states. Lower panel: An example of APSD and CPSD spectra for Acq12 and the fitted Lorentzian curve marked by solid black line. The spectra were calculated using $N=2000$ and $d t=1.02 \mathrm{~ms}$.

\subsection{The numerical parameters}

Power spectral density methods inevitably introduce additional purely numerical parameters. Unlike physical parameters of the experimental system, these parameters should have little or no effect on the results of the analysis. The acquisition method of time stamps records the time of each detection within a resolution of $25 \mathrm{~ns}$. This time resolution is too fine and not adequate for power spectral density calculations due to the relevant signal bandwidth of 1-80 Hz. Therefore, the detector signal is binned on a coarser time resolution. Furthermore, the power spectral density is not calculated for the entire signal at once due to computer memory limitations. The long signal is divided into shorter segments (or buffers), each of duration 


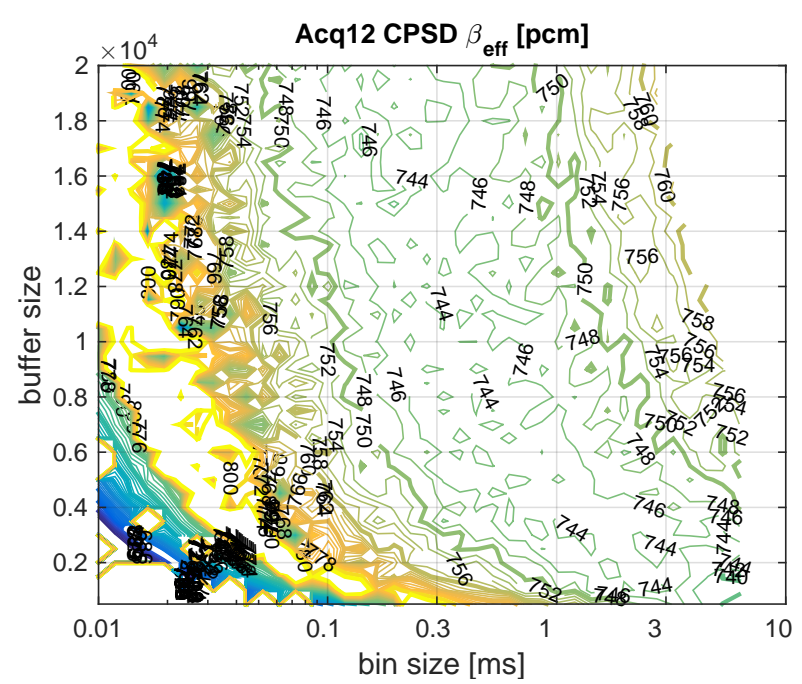
as The results for the critical state Acq12 are shown in Fig. 4.

$T=N d t$, where $d t$ is the size of the time bin and $N$ is the number of bins (buffer size) considered for a single spectrum calculation. For each segment, the power spectral density (CPSD or APSD) is calculated and averaged with the rest of the spectra calculated for the other segments.

This standard calculation method introduces two numerical, not physical, parameters into the procedure, i.e., the buffer size $N$ and the time bin size $d t$. Hence, the discrete form of the PSD should be written

$$
\mathrm{PSD} \equiv \operatorname{PSD}(\omega ; N, d t)
$$

The explicit dependence of the CPSD and APSD on these parameters is demonstrated in Eqs. (2)-(6). In order to evaluate the sensitivity of the calculated kinetics parameters $\beta_{\text {eff }}$ and $\Lambda$ to these numerical parameters, the calculation procedure is repeated using the same data but with different numerical parameters.

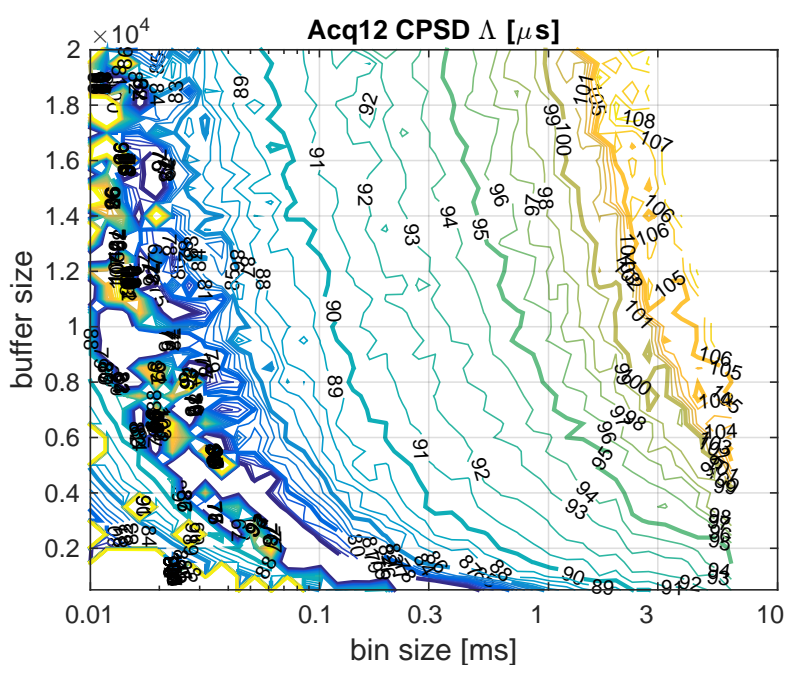

Figure 4: Sensitivity of the obtained effective delayed neutron fraction $\beta_{\text {eff }}$ and the prompt neutron generation time $\Lambda$ to the buffer size $N$ and the time bin size $d t$ for the critical state Acq12 using CPSD spectra.

It is clear from Fig. 4 that the numerical parameters $N$ and $d t$ have a pronounced effect on the obtained kinetic parameters. Moreover, methodologies for tuning these parameters are not usually addressed. Initially, no compelling physical arguments favoring a specific set of values for the buffer size $N$ and the time bin size $d t$ were found. These parameters are usually set such that the sensitivity of the obtained results is minimized and the residuals are normally distributed without any trend at low or high frequencies. Examination of Fig. 4 indeed reveals areas in the numerical parameters space where the value of $\beta_{\text {eff }}$ is only weakly sensitive to the parameters values, but no such areas are found for $\Lambda$. The upper right corner is empty since the signals recorded by the acquisition system for the critical state Acq12 were segmented into files containing 55 seconds each, hence no spectra were generated for $N d t>55$ seconds. 


\subsection{The relevant frequency range}

The irregular and erratic behavior of the fit results in the left and lower left parts of the parameter space (mainly small $d t$ ) is due to the fact that the relevant frequency range, where the PSD possesses physical meaning (i.e., the transfer function and not white noise), is roughly between 1-80 Hz (Geslot et al., 2015). This range depends of course on the physical properties of the specific core and can assume different values. Moreover, the kinetic parameters presented in Fig. 4 are fitted over the entire spectrum and not confined to some predefined frequency range.

The buffer size $N$ and the time bin size $d t$ determine the frequency range and resolution of the derived spectra. The maximal frequency is determined by the Nyquist frequency, $f_{\max }=\frac{1}{2 d t}$, and the minimal frequency, which is equivalent to the spectrum resolution, is determined according to $d f=\frac{1}{T}=\frac{1}{N d t}$. Hence, the requirement $f_{\max } \geqslant 80 \mathrm{~Hz}$ means $d t \leqslant \frac{1}{160} \mathrm{~s}$ and $f_{\min } \leqslant 1 \mathrm{~Hz}$ means $N d t \geqslant 1 \mathrm{~s}$. The values of $f_{\min }$ as a function of $d t$ and $N$ are illustrated in Fig. 5 , where the line $d t=\frac{1}{160} \mathrm{~s}$ is also marked. Any pair of $d t$ and $N$ which define a frequency range that includes the range 1-80 Hz should be considered as relevant for PSD fit procedures.

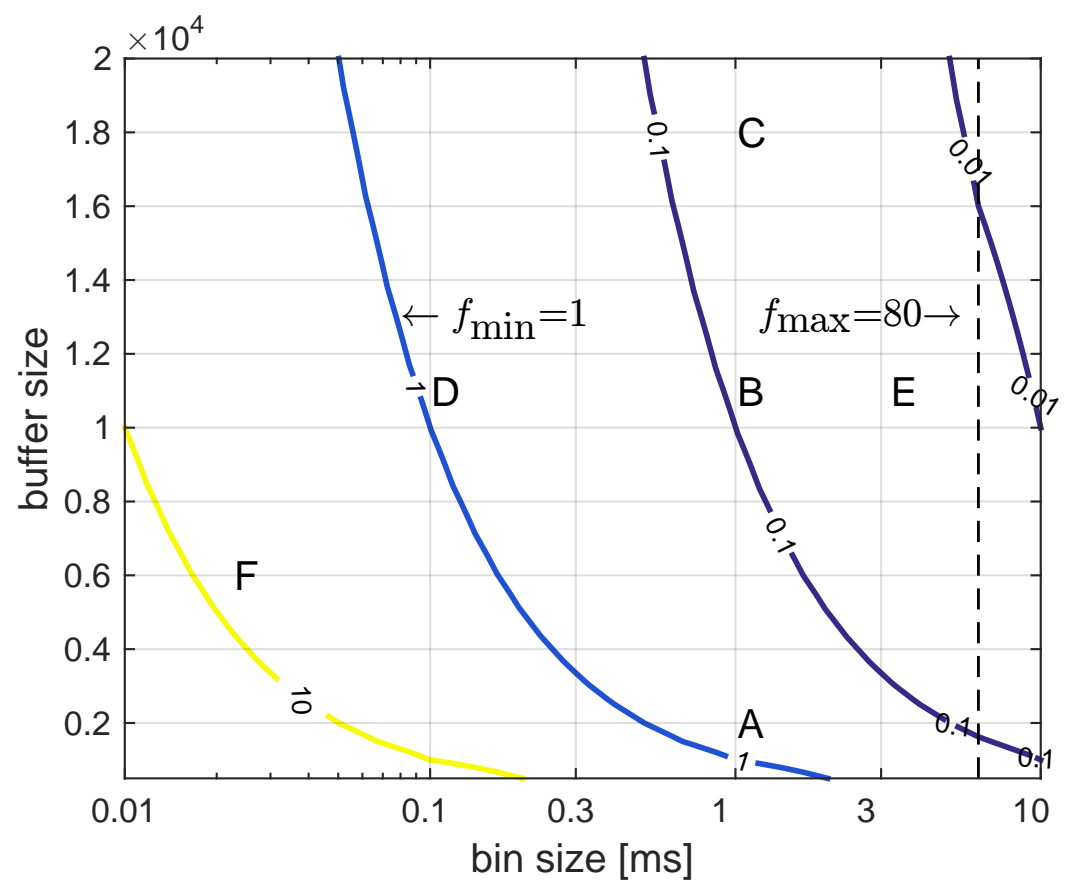

Figure 5: The values of $f_{\min }$ as a function of $d t$ and $N$. The line $d t=\frac{1}{160} \mathrm{~s}$ is marked with dashed black line. Points A-E represent appropriate sets of values $(d t, N)$, i.e., the PSD range spans the $1-80 \mathrm{~Hz}$ range. Point $\mathrm{F}$ represent inappropriate set of values for PSD fit.

Points A-E in Fig. 5 represent sets of values $(d t, N)$ appropriate for PSD fit, i.e., the PSD range includes the 1-80 Hz range. Point $\mathrm{F}$ represent inappropriate set of values for PSD fit. The parameters of points A-F 
are given in Table 2 and the corresponding spectra and Lorentzian fits are shown in Fig. 6.

Table 2: Parameters of points A-F in Fig. 5.

\begin{tabular}{cccccc}
\hline \hline Point & $d t[\mathrm{~ms}]$ & $N$ & $f_{\text {min }}-f_{\max }[\mathrm{Hz}]$ & $\beta_{\mathrm{eff}}[\mathrm{pcm}]$ & $\Lambda[\mu \mathrm{s}]$ \\
\hline \hline A & $1.02 \mathrm{e}-03$ & 2000 & $4.92 \mathrm{e}-01-4.92 \mathrm{e}+02$ & 744 & 91 \\
$\mathrm{~B}$ & $1.02 \mathrm{e}-03$ & 11000 & $8.94 \mathrm{e}-02-4.92 \mathrm{e}+02$ & 747 & 97 \\
$\mathrm{C}$ & $1.02 \mathrm{e}-03$ & 18000 & $5.46 \mathrm{e}-02-4.92 \mathrm{e}+02$ & 750 & 99 \\
$\mathrm{D}$ & $1.01 \mathrm{e}-04$ & 11000 & $9.01 \mathrm{e}-01-4.96 \mathrm{e}+03$ & 751 & 90 \\
$\mathrm{E}$ & $3.23 \mathrm{e}-03$ & 11000 & $2.82 \mathrm{e}-02-1.55 \mathrm{e}+02$ & 755 & 105 \\
$\mathrm{~F}$ & $2.28 \mathrm{e}-05$ & 6000 & $7.30 \mathrm{e}+00-2.19 \mathrm{e}+04$ & 869 & 106 \\
\hline \hline
\end{tabular}

The striking observation from Fig. 6 and Table 2 is that all points A-E cover very well the transition section of the transfer function, i.e., the relevant bandwidth 1-80 Hz, and they all exhibit excellent fits with uniform distribution of the normalized residuals. Hence, all these point are appropriate for PSD analysis and derivation of the kinetic parameters. However, as shown in Table 2, the obtained values of the kinetic parameters vary significantly between the different points, where no point is favored over the next one.

Once we have established some guide rules for selecting proper sets of $(d t, N)$ values, Fig. 4 is redrawn in Fig. 7 only for appropriate parameters which enable proper fit procedure.

This representation of the sensitivity of the obtained kinetic parameters is much more insightful since although any point in Fig. 7 is legitimate for the Lorentzian fit procedure, the variance in the obtained results is significant. For example, Geslot et al. (2015) calculated the PSD using time resolution of $1 \mathrm{~ms}$ and frequency resolution of $0.5 \mathrm{~Hz}$, which correspond to $d t=1 \mathrm{~ms}$ and $N=2000$. Looking at Fig. 7, this point is part of a large set of equivalent points where none are physically favored, but produce different results.

\subsection{Quantifying the uncertainty}

One possible course of action in determining the value of $\beta_{\text {eff }}$ and $\Lambda$ is to average their values in the relevant frequency area, since they are all physically equivalent with appropriate spectrum for fit. The standard deviation over this set will give a measure of the uncertainty originating from the choice of numerical parameters such as $d t$ and $N$. In the case of CPSD analysis of the critical state Acq12 shown in Fig. 7, the mean and standard deviation are $\beta_{\mathrm{eff}}=756.7 \pm 3.8 \mathrm{pcm}$ and $\Lambda=91.7 \pm 3.6 \mu \mathrm{s}$. It should be noted that in this paper, the precise and exact values of the kinetic parameters are of less importance or experimental validity (this point is further discussed in later sections). Instead, the important result is the estimation of the associated uncertainty and the fact that it is of significant magnitude compared to other uncertainties and should not be ignored in the future. 

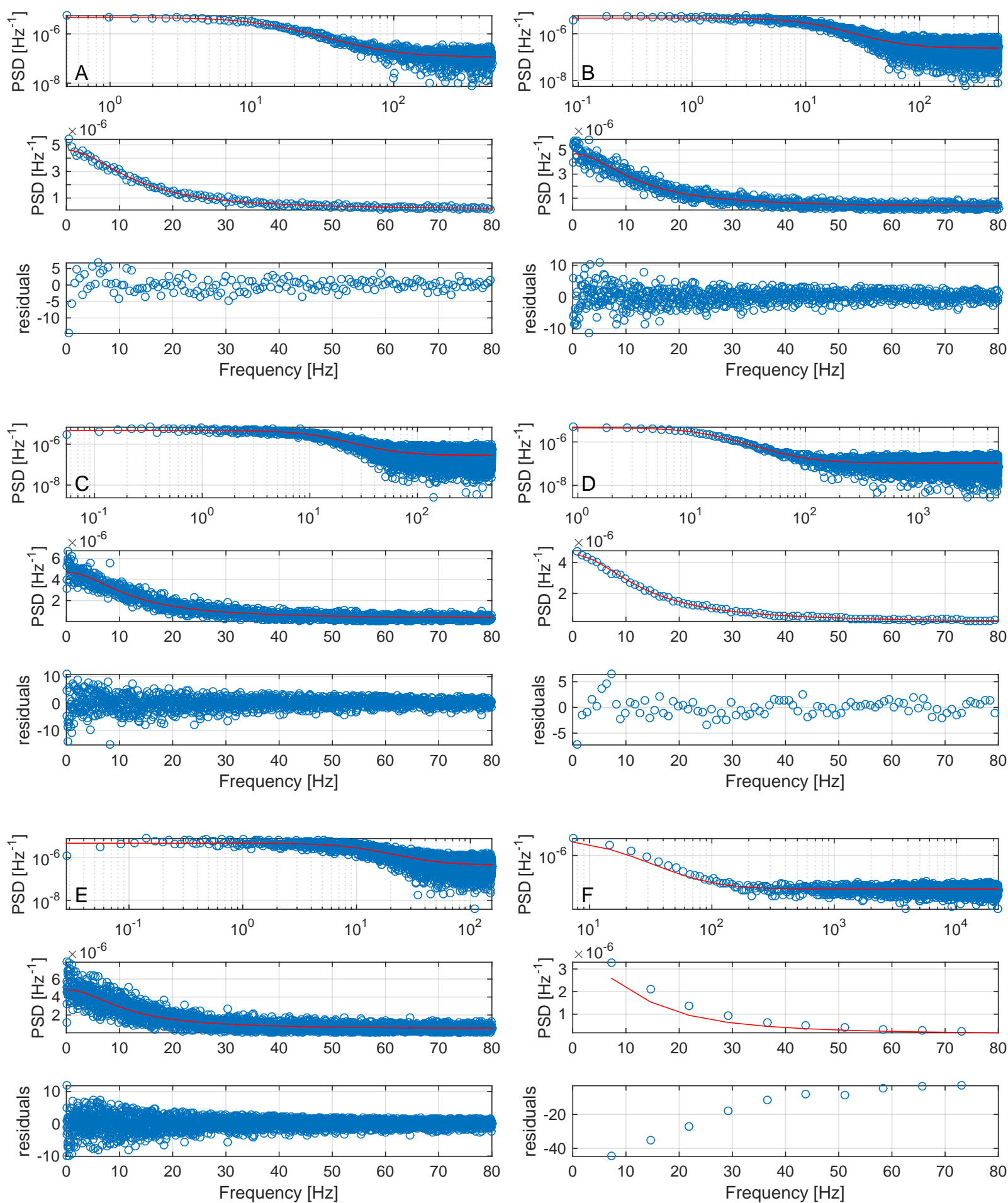

Figure 6: Full spectra (upper panel), Lorentzian fit on the range 1-80 Hz (middle panel), and the normalized residuals (lower panel) for the points A-F detailed in Table 2 and shown in Fig. 5. 

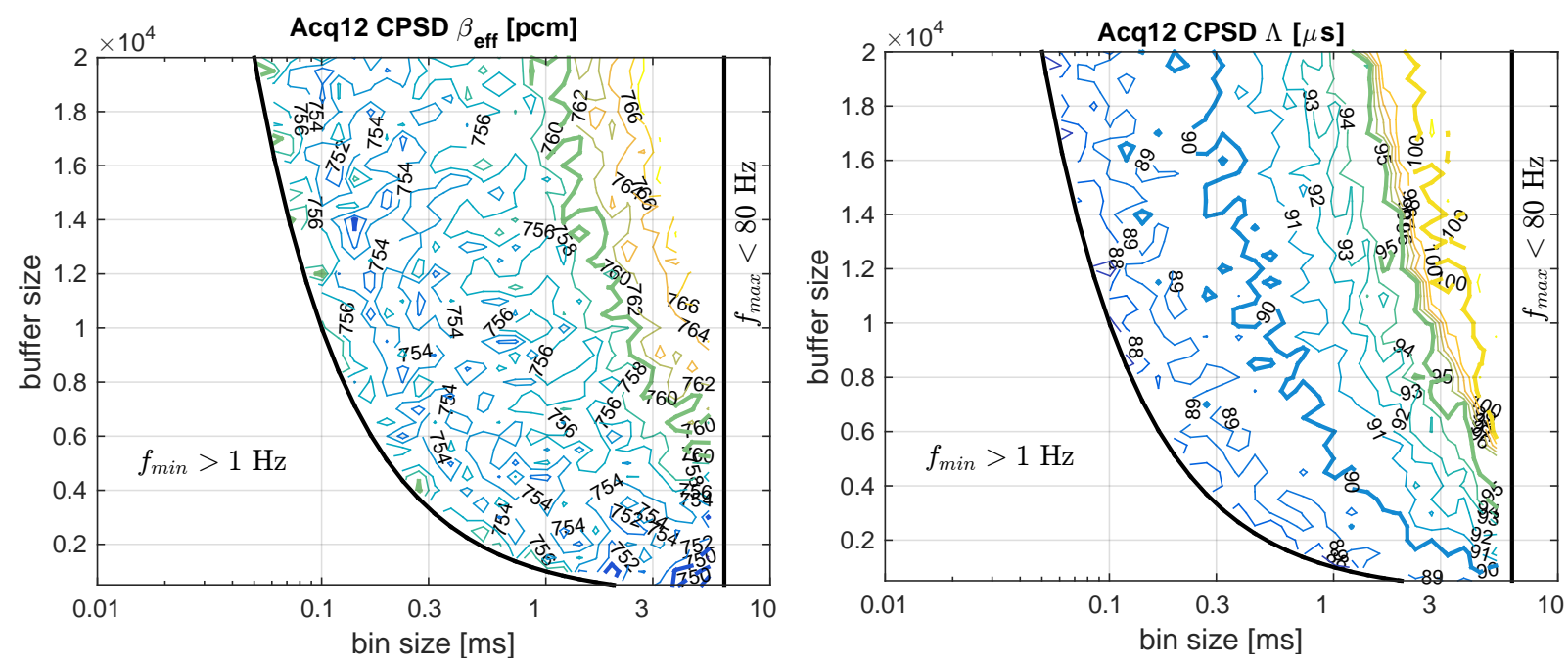

Figure 7: Sensitivity of the obtained kinetic parameters to the buffer size $N$ and the time bin size $d t$ over the relevant frequency range for the critical state Acq12 using CPSD spectra.

\subsection{CPSD results for subcritical states}

The CPSD analysis described in section 4 was also applied to the two subcritical states Acq16 and Acq19. The results are shown in Fig. 8 and show similar qualitative behavior of the kinetic parameters sensitivity to the choice of $d t$ and $N$. Quantitatively, however, the uncertainties are smaller compared to the critical state analysis (Acq12), and the mean and standard deviation are $\beta_{\text {eff }}=734.4 \pm 4.4 \mathrm{pcm}$ and $\Lambda=91.6 \pm 3.0$ $\mu \mathrm{s}$ for Acq16 and $\beta_{\mathrm{eff}}=715.4 \pm 3.1 \mathrm{pcm}$ and $\Lambda=89.6 \pm 3.2 \mu \mathrm{s}$ for Acq19.

EREZ: Moreover, comparing to Fig. 7, it seems that the sensitivity of the CPSD to the numerical parameters is not only weaker in subcritical states than in the critical state, but also that the kinetic parameters exhibit smoother and more homogeneous behavior over the parameter space. This could be related to the fact that the statistical errors associated with higher moments of the count rate (used in estimators like CPSD, APSD, Fyenman- $\alpha$, etc.) converge faster for subcritical measurements than for critical ones. As a general rule, the convergence rate of the variance of higher moments is proportional to the inverse of the reactivity. More specifically, the statistical variance of moment $M_{n}$ of order $n$ converges at a rate inversely proportional to the reactivity to the power of $2 n$, i.e., $\operatorname{Var}\left(M_{n}\right) \sim \frac{1}{\rho^{2 n}}$ (Dubi and Kolin, 2016).

\subsection{APSD results}

An APSD analysis was carried out on all three reactivity states along the guidelines that were phrased in section 4 regarding the relevant range of numerical parameters to be used for fit procedure. The sensitivity of the obtained kinetics parameters to the choice of $d t$ and $N$ show similar qualitative behavior as exhibited 

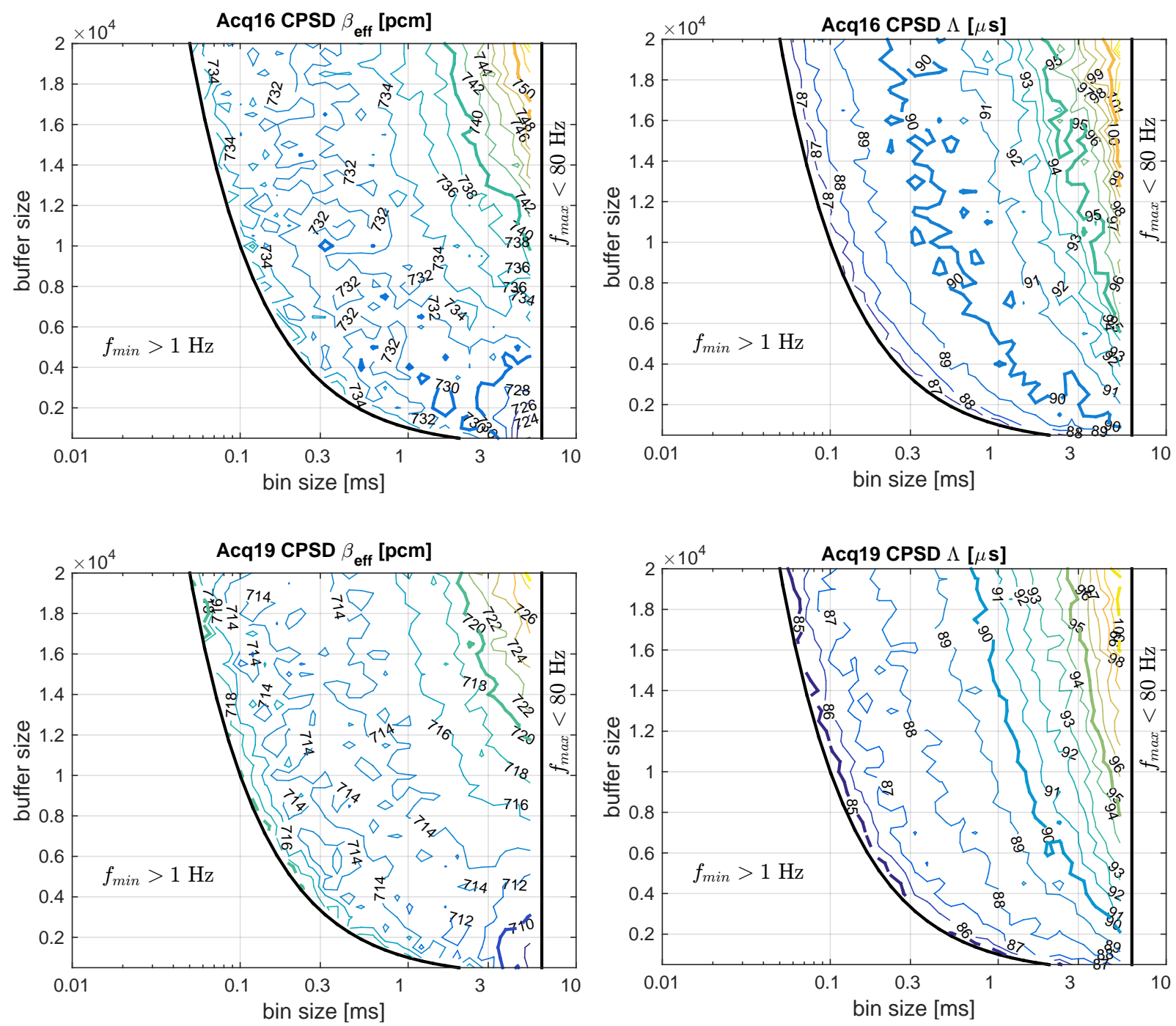

Figure 8: Sensitivity of the obtained kinetic parameters to the buffer size $N$ and the time bin size $d t$ over the relevant frequency range for the subcritical states Acq16 and Acq19 using CPSD spectra. 

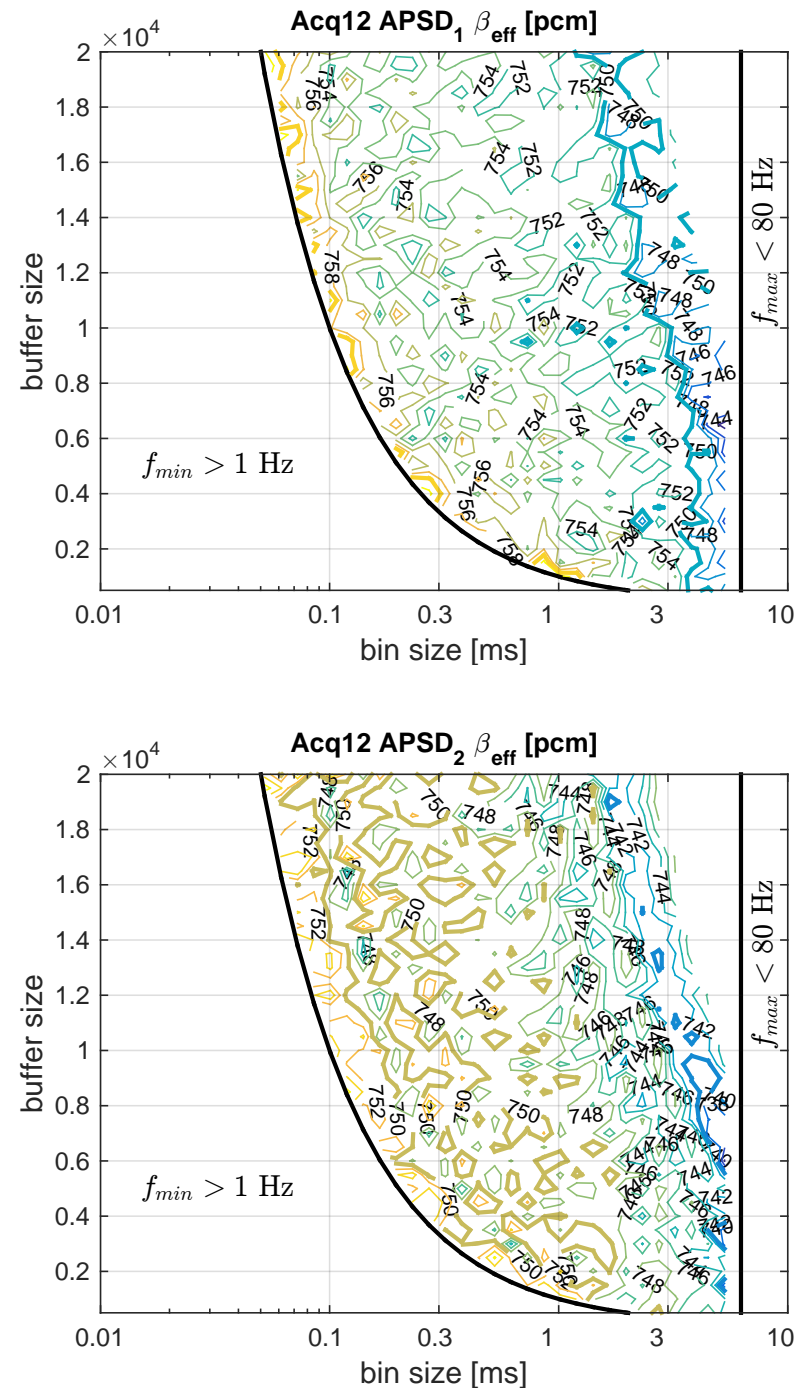
state Acq12.
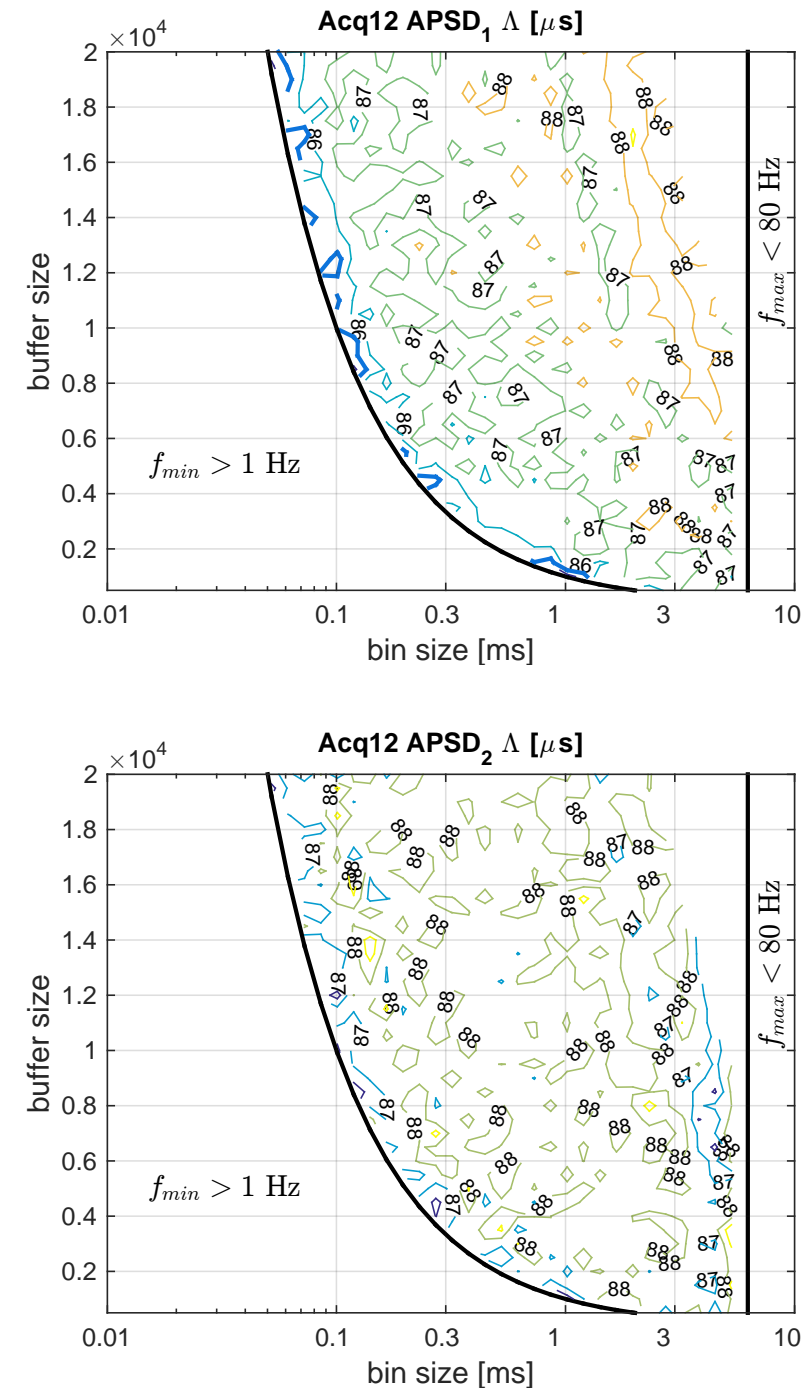

Figure 9: Sensitivity of the obtained kinetic parameters to the buffer size $N$ and the time bin size $d t$ over the relevant frequency range for the critical state Acq12 using $\mathrm{APSD}_{1}$ (upper panels) and $\mathrm{APSD}_{2}$ (lower panels) spectra.

in the CPSD analysis. An example is shown in Fig. 9 for both $\mathrm{APSD}_{1}$ and $\mathrm{APSD}_{2}$ analysis of the critical 
other sources of uncertainty.

Table 3: Mean and standard deviation $(1 \sigma)$ of the kinetic parameters values over the relevant frequency range for both CPSD and APSD analysis of three reactivity states. The emphasized values are the mean and RMS of the different CPSD/APSD results.

\begin{tabular}{|c|c|c|c|c|c|}
\hline \multirow{2}{*}{ Reactivity state } & \multirow{2}{*}{ PSD method } & \multicolumn{2}{|c|}{ Current work } & \multicolumn{2}{|c|}{ Geslot et al. (2015) } \\
\hline & & $\beta_{\text {eff }}[\mathrm{pcm}]$ & $\Lambda[\mu \mathrm{s}]$ & $\beta_{\text {eff }}[\mathrm{pcm}]$ & $\Lambda[\mu \mathrm{s}]$ \\
\hline \multirow{4}{*}{ Acq12 } & CPSD & $756.7 \pm 3.8$ & $91.7 \pm 3.6$ & $746.8 \pm 1.8$ & $94.5 \pm 0.7$ \\
\hline & $\mathrm{APSD}_{1}$ & $753.2 \pm 3.3$ & $87.1 \pm 0.8$ & $750.4 \pm 2.8$ & $94.8 \pm 1.3$ \\
\hline & $\mathrm{APSD}_{2}$ & $748.0 \pm 3.7$ & $87.7 \pm 0.6$ & $749.1 \pm 2.4$ & $93.7 \pm 1.1$ \\
\hline & & $752.6 \pm 3.6$ & $88.8 \pm 2.2$ & $748.8 \pm 2.4$ & $94.3 \pm 1.1$ \\
\hline \multirow{4}{*}{ Acq16 } & CPSD & $734.4 \pm 4.4$ & $91.6 \pm 3.0$ & - & - \\
\hline & $\mathrm{APSD}_{1}$ & $769.0 \pm 4.9$ & $93.4 \pm 1.3$ & - & - \\
\hline & $\mathrm{APSD}_{2}$ & $683.6 \pm 4.4$ & $86.8 \pm 0.9$ & - & - \\
\hline & & $729.0 \pm 4.6$ & $90.4 \pm 2.0$ & - & - \\
\hline \multirow{4}{*}{ Acq19 } & CPSD & $715.4 \pm 3.1$ & $89.6 \pm 3.2$ & - & - \\
\hline & $\mathrm{APSD}_{1}$ & $724.5 \pm 3.2$ & $89.1 \pm 1.0$ & - & - \\
\hline & $\mathrm{APSD}_{2}$ & $694.3 \pm 2.8$ & $84.0 \pm 0.9$ & - & - \\
\hline & & $711.4 \pm 3.0$ & $87.6 \pm 2.0$ & - & - \\
\hline
\end{tabular}

EREZ: The discrepancies of the results is two fold. First, the CPSD and the APSD results are in well agreement for the critical state Acq12, and also agree well with the results obtained by Geslot et al. (2015) for $\beta_{\text {eff }}$ (but less for $\Lambda$ ). However, the discrepancies between the results associated separately with each detector, i.e., $\mathrm{APSD}_{1}$ and $\mathrm{APSD}_{2}$, increase as the core becomes more subcritical. Generally, results obtained using counts from detector 1 clearly exceed the results obtained using counts from detector 2 for both $\beta_{\text {eff }}$ and $\Lambda$. This disagreement was also observed by Gilad et al. (2016), where the subcritical states were analyzed using a completely different method, i.e., the Feynman-Y method. Second, it seems that the subcriticality level of the core during the experiment significantly influences the results and neither the CPSD nor the APSD methods produce consistent results for the kinetic parameters by analyzing the different reactivity states. EREZ: Several possible sources for the dispersion of the results from the two detectors comes to mind. The detection efficiency is different between the two detectors, which lead to small discrepancies between the statistical characteristics of their associated neutron counts. Although these discrepancies a small, 
the fact that no dead-time correction was applied to any of the detectors' counts may increase the observed inconsistency (the CPSD is somewhat less sensitive to dead-time corrections than APSD). Moreover, different geometrical positions of the detectors may give rise to small spatial effects. Finally, inconsistencies in the evaluation of the subcriticality levels of the different states (as suggested by Gilad et al. (2015)) or in the evaluation of the integral fission rates can bear significant deviations in the obtained kinetic parameters.

EREZ: The dispersion of the results is important, real, and no obvious trend can be identified, which makes the use of average results a bit unreliable. Having said that, a thorough analysis of these discrepancies is beyond the scope of this paper, which focuses more on the numerical uncertainties associated with the PSD techniques and less with the absolute values of the obtained kinetic parameters.

\section{Conclusions}

Power spectral density methods (Cohn- $\alpha$ methods) are well-known and widely used for the analysis of neutron noise experiments and obtaining the reactor core integral kinetic parameters, i.e. the effective delayed neutron fraction $\beta_{\text {eff }}$ and the prompt neutron generation time $\Lambda$. The Cohn- $\alpha$ methods are considered as the standard data processing procedure in the case of a current acquisition system that works at high fission rates. These methods have proved to be very robust and produced minimum uncertainties.

Uncertainty analysis of Cohn- $\alpha$ techniques usually considers important source for uncertainty, e.g., statistical fluctuations in the neutron count, power drifts, uncertainties in the Diven factor, the integral fission rate, and in the reactivity value. These uncertainties are then properly propagated through the Cohn- $\alpha$ procedure in order to evaluate the total uncertainty in the obtained kinetic parameters. For example, the analysis by Geslot et al. (2015) on the same data for the critical state using Power Spectral Density (PSD) techniques leads to uncertainties of 1.8-2.8 pcm in the value of $\beta_{\text {eff }}$ and $0.7-1.3 \mu \mathrm{s}$ in $\Lambda$ (at $1 \sigma$ ).

However, the uncertainty associated with the numerical parameters used in the power spectra calculation procedure, e.g., time bin size and buffer size, is hardly discussed in the literature and generally overlooked, whereas these parameters are often determined rather arbitrarily according to the acquisition system technical specifications. Moreover, well-defined criteria or methodologies for evaluating their associated uncertainties are not addressed.

In this paper, The Cohn- $\alpha$ method is implemented to analyze critical and subcritical configurations of the MAESTRO core in the MINERVE zero power reactor in order to measure its integral kinetic parameters, i.e. effective delayed neutron fraction $\beta_{\text {eff }}$ and the prompt neutron generation time $\Lambda$.

The sensitivity of the obtained kinetic parameters to the choice of numerical parameters used for spectra calculations is studied and found to be pronounced. Examination of this sensitivity (Fig. 4) reveals extremely sensitive and erratic behavior of the fit results for small $d t$ and a wide range of $N$ values due to improper frequency range for the PSD, i.e., the PSD does not contain the physically viable frequency range of the 
zero power transfer function, which is estimated roughly to be between 1-80 Hz for the MAESTRO core configuration.

This extremely sensitive and erratic behavior is eliminated once the numerical parameter space $(d t, N)$ is restricted to values which include the proper frequency range for the PSD. However, although the sensitivity of the obtained kinetic parameters to the numerical parameters is reduced dramatically, it does not become negligible and show pronounce changes over the $(d t, N)$ space (Figs. 7, 8, 9).

EREZ: It should be noted that the choice to fit the Lorentzian curve using spectra in the range 1-80 $\mathrm{Hz}$, although based on physical considerations, is rather arbitrary and this arbitrariness is inflicted on the uncertainty. This frequency range should be set according to the form of the reactor's transfer function and the signal-to-noise ratio. The sensitivity of the obtained kinetic parameters to the fitting range was superficially examined by using the range 1-120 Hz for comparison, which yielded no significant differences.

EREZ: Essentially, the different spectra, which are derived from the same measured data (e.g., Acq12, Acq16, or Acq19) using different sets of numerical parameters $(d t, N)$, encapsulate the same amount of information. The different choices of numerical parameters simply distribute this information differently in the spectra. Large buffers (large $N$ ) lead to finer resolution in the frequency domain but less statistics on each point, whereas small buffers lead to coarser spectral resolution but better statistics on each point. This trade-off affect the fit procedure, as nicely demonstrated by considering points A (small buffer) and C (large buffer) in Figs. 5 and 6.

A novel methodology is proposed for analyzing the kinetic parameters' sensitivity to the power spectra calculations and for quantifying the associated uncertainty. Since any point in the numerical parameter space that satisfies the requirements for physically viable frequency range (Fig. 5) is adequate for Lorentzian fit, the values of the kinetic parameters and the associated uncertainty are determined by the mean and standard deviation of these parameters over the proper numerical parameter space. It should be noted that the fit results exhibit rather smooth and robust behavior over the numerical parameter space.

The uncertainties originate from the sensitivity of the kinetic parameters to the numerical parameters used for PSD calculation are summarized in Table 3. The uncertainty value for the critical state (Acq12) in $\beta_{\text {eff }}$ is $3.8 \mathrm{pcm}$ for CPSD and $\sim 3.5 \mathrm{pcm}$ for APSD analyses, and in $\Lambda$ is $3.6 \mu$ s for CPSD and $\sim 0.7 \mu$ s for APSD analyses. These values are significant and non-negligible comparing to the corresponding 1.8-2.8 pcm and 0.7-1.3 $\mu$ s uncertainty values calculated by Geslot et al. (2015), where the PSD spectra were calculated at a single point in the $(d t, N)$ space, i.e., $d t=1 \mathrm{~ms}$ and $N=2000$.

EREZ: The discrepancies between the results associated separately with each detector increase as the core becomes more subcritical and results obtained using counts from detector 1 clearly exceed the results obtained using counts from detector 2 for both $\beta_{\text {eff }}$ and $\Lambda$. This disagreement was also observed by Gilad et al. (2016), where the subcritical states were analyzed using the Feynman-Y method, which is different 
from PSD methods in that it is not based on the reactor transfer function. Several possible sources for the dispersion of the results from the two detectors are discussed in section 4.5, e.g. the absence of dead-time cOs correction, spatial effects, inconsistencies in the evaluation of the subcriticality levels and in the evaluation of the integral fission rates. Although the dispersion of the results is important and real, a thorough analysis of these discrepancies is beyond the scope of this paper.

We conclude by stating that the uncertainties in the kinetic parameters $\left(\beta_{\text {eff }}\right.$ and $\left.\Lambda\right)$ calculated using Cohn- $\alpha$ methods, which are associated with the numerical parameters time bin size and buffer size used for spectra calculations, are significant and should not be neglected. 


\section{References}

Cacuci, D. G., 2010. Handbook of Nuclear Engineering. Springer.

Carre, J., Oliveira, J. D. C., 1975. Measurements of kinetic parameters by noise techniques on the MINERVE reactor. Annals of Nuclear Energy 2 (2), 197- 206.

Cohn, C. E., 1960. A simplified theory of pile noise. Nuclear Science and Engineering 7, 472-475.

de Izarra, G., Jammes, C., Geslot, B., Di Salvo, J., Destouches, C., 2015. SPECTRON, a neutron noise measurement system in frequency domain. Review of Scientific Instruments 86 (11), 115111.

URL http://scitation.aip.org/content/aip/journal/rsi/86/11/10.1063/1.4935250

Diniz, R., dos Santos, A., 2002. A noise analysis approach for measuring the decay constants and the relative abundance of delayed neutrons in a zero power critical facility. Journal of Nuclear Science and Technology Sup2, 669-672.

Diven, B. C., Martin, H. C., Taschek, R. F., Terrell, J., 1956. Multiplicities of Fission Neutrons. Phys. Rev. 101, $1012-1015$. dos Santos, A., Diniz, R., Fanaro, L. C., Jerez, R., de Andrade e Silva, G. S., Yamaguchi, M., 2006. A proposal of a benchmark for beff, beff/l, and 1 of thermal reactors fueled with slightly enriched uranium. Annals of Nuclear Energy 33 (9), 848 - 855 . URL http://www.sciencedirect.com/science/article/pii/S0306454906000600

Dubi, C., Kolin, A., 2016. Analytic derivation of the statistical error in the Feynman- method. Annals of Nuclear Energy 88, $186-193$.

URL http://www.sciencedirect.com/science/article/pii/S0306454915005277

Geslot, B., Gruel, A., Pepino, A., Salvo, J. D., de Izarra, G., Jammes, C., Destouches, C., Blaise, P., 2015. Pile noise experiment in MINERVE reactor to estimate kinetic parameters using various data processing methods. In: 4th ANIMMA, 20-24 April 2015, Lisbon.

Gilad, E., Kolin, A., Rivin, O., Dubi, C., Geslot, B., Blaise, P., 2016. Analysis of critical and subcritical neutron noise experiments in MINERVE using advanced noise techniques. In: PHYSOR 2016: Unifying Theory and Experiments in the 21st Century, May 1-5, 2016, Sun Valley, Idaho, USA.

Gilad, E., Rivin, O., Ettedgui, H., Yaar, I., Geslot, B., Pepino, A., Salvo, J. D., Gruel, A., Blaise, P., 2015. Experimental estimation of the delayed neutron fraction of the MAESTRO core in the MINERVE zero power reactor. Journal of Nuclear Science and Technology 52 (7-8), 1026-1033.

Keepin, G. R., 1965. Physics of Nuclear Kinetics. Addison-Wesley.

Leconte, P., Geslot, B., Gruel, A., Pepino, A., Derriennic, M., Di-Salvo, J., Antony, M., Eschbach, R., Cathalau, S., 2013. MAESTRO: An ambitious experimental programme for the improvement of nuclear data of structural, detection, moderating and absorbing materials - First results for nat V, 55Mn, 59Co and 103Rh. In: 3rd ANIMMA, 23-27 June 2013, Marseille, France.

pascal Hudelot, J., Klann, R., Fougeras, P., Jorion, F., Drin, N., Donnet, L., 2004. OSMOSE: An Experimental Program for the Qualification of Integral Cross Sections of Actinides. In: PHYSOR 2004, Chicago, Illinois, April 25-29.

Perret, G., 2015. Delayed neutron fraction and prompt decay constant measurement in the MINERVE reactor using the PSI instrumentation. In: 4th ANIMMA, 20-24 April 2015, Lisbon.

Santamarina, A., Blaise, P., Erradi, L., Fougeras, P., 2012. Calculation of LWR beff kinetic parameters: Validation on the MISTRAL experimental program. Annals of Nuclear Energy 48, 51-59.

Williams, M. M. R., 1974. Random Processes in Nuclear Reactors. Pergamon Press. 